\title{
APC2 and Axin promote mitotic fidelity by facilitating centrosome separation and cytoskeletal regulation
}

\author{
John S. Poulton ${ }^{1,2}$, Frank W. Mu' ${ }^{1}$, David M. Roberts ${ }^{3}$ and Mark Peifer ${ }^{1,2, *}$
}

\begin{abstract}
SUMMARY
To ensure the accurate transmission of genetic material, chromosome segregation must occur with extremely high fidelity. Segregation errors lead to chromosomal instability (CIN), with deleterious consequences. Mutations in the tumor suppressor adenomatous polyposis coli $(A P C)$ initiate most colon cancers and have also been suggested to promote disease progression through increased CIN, but the mechanistic role of APC in preventing CIN remains controversial. Using fly embryos as a model, we investigated the role of APC proteins in CIN. Our findings suggest that APC2 loss leads to increased rates of chromosome segregation error. This occurs through a cascade of events beginning with incomplete centrosome separation leading to failure to inhibit formation of ectopic cleavage furrows, which result in mitotic defects and DNA damage. We test several hypotheses related to the mechanism of action of APC2, revealing that $A P C 2$ functions at the embryonic cortex with several protein partners, including Axin, to promote mitotic fidelity. Our in vivo data demonstrate that APC2 protects genome stability by modulating mitotic fidelity through regulation of the cytoskeleton.
\end{abstract}

KEY WORDS: APC, Axin, Drosophila, Chromosome segregation, Cytoskeleton, Genome stability

\section{INTRODUCTION}

The tumor suppressor adenomatous polyposis coli (APC) has dual roles in regulating Wnt signaling and the cytoskeleton (Fearon, 2011). In its cytoskeletal roles, APC is suggested to regulate mitosis. During mitosis, chromosomes must be accurately segregated into daughter cells with extremely high fidelity. Mitotic fidelity is rooted in formation of bipolar spindles via microtubule nucleation by centrosomes (Tanenbaum and Medema, 2010). As spindles form, microtubules must properly attach to kinetochores to facilitate chromosome segregation.

Despite the many mitotic regulators and checkpoints, errors inevitably occur. In normal cells in vitro, chromosome missegregation occurs in $\sim 1 \%$ of divisions, leading to aneuploidy (Cimini et al., 1999; Thompson and Compton, 2008). Missegregation can be caused by defects at several steps during mitosis, e.g. abnormal centrosome number, function or separation can disrupt bipolar spindles (Silkworth et al., 2012; Tanenbaum and Medema, 2010). Aneuploidy has deleterious effects on cell behavior (Gordon et al., 2012), and chromosome mis-segregation can also lead directly to DNA damage, further destabilizing the genome (Crasta et al., 2012; Janssen et al., 2011).

Chromosome mis-segregation plays a crucial role in the multistep process leading to aggressive cancers (Gordon et al., 2012). Mutations in tumor suppressors and proto-oncogenes promote cancer initiation by misregulating signaling pathways, driving the cell cycle. However, these initial mutations are not sufficient; instead, accumulation of additional genetic changes leads to metastatic tumors. It has been recognized for more than a century that cancer cells have a highly elevated frequency of chromosome segregation defects. Chromosomal instability (CIN) is a hallmark of

\footnotetext{
${ }^{1}$ Department of Biology, University of North Carolina, Chapel Hill, NC 27599, USA. ${ }^{2}$ Lineberger Comprehensive Cancer Center, University of North Carolina, Chapel Hill, NC 27599, USA. ${ }^{3}$ Department of Biology, Franklin and Marshall College, Lancaster, PA 17604, USA.

*Author for correspondence (peifer@unc.edu)
}

Accepted 30 July 2013 advanced tumors (Hanahan and Weinberg, 2011), and leads to a cycle of mitotic defects, further destabilizing the genome and promoting cancer progression.

More than $80 \%$ of colorectal tumors are initiated by loss-offunction mutations in the tumor suppressor APC (Fearon, 2011). APC prevents tumor initiation by negatively regulating Wnt signaling, helping target $\beta$ catenin ( $\beta$ cat) for destruction. APC loss activates Wnt signaling, with profound consequences for proliferation and differentiation (Phelps et al., 2009; Smits et al., 1999). However, there is also evidence that APC loss promotes tumor progression. Approximately $80 \%$ of colorectal tumors have some form of CIN (Migliore et al., 2011; Rajagopalan et al., 2003; Shih et al., 2001). APC disruption has been linked to increased CIN, providing a mechanism by which it may drive tumor progression (Alberici and Fodde, 2006). Consistent with this hypothesis, many $A P C$ mutant colon cancer cell lines display high-level CIN, whereas a colon cancer line with an oncogenic $\beta$ cat mutation but wild-type APC is chromosomally stable (Caldwell et al., 2007; Lengauer et al., 1997; Thompson and Compton, 2008). Furthermore, one study found $\sim 60 \%$ of polyps from patients with inherited $A P C$ mutations contain aneuploid cells, suggesting that CIN might precede accumulation of additional mutations (Cardoso et al., 2006), although others found no significant increase in CIN in adenomas (Haigis et al., 2002; Sieber et al., 2002). These data suggest a role for $\mathrm{APC}$ in $\mathrm{CIN}$ prevention.

APC proteins are multidomain scaffolds that interact with both the actin and microtubule cytoskeletons (Näthke, 2006). Although many studies suggest that $\mathrm{CIN}$ results from disrupting the cytoskeletal functions of APC, the suggested mechanisms are diverse. They include proposed roles in microtubule-kinetochore attachment (Fodde et al., 2001; Kaplan et al., 2001), microtubule/spindle dynamics (Bahmanyar et al., 2009; Caldwell et al., 2007; Dikovskaya et al., 2004; Green et al., 2005) and the spindle assembly checkpoint (Dikovskaya et al., 2007; Radulescu et al., 2010); others suggested indirect effects via misregulated Wnt signaling (Aoki et al., 2007; Hadjihannas et al., 2006). Thus, although most studies support a role for APC in preventing CIN, its mechanism of action remains unclear. Furthermore, most studies 
were in cultured cells, in which normal mechanisms protecting genome stability may be weakened (Farrell et al., 1991; Mazars et al., 1992), and those performed in vivo utilized truncated APC alleles that can be dominant-negative (Cheung et al., 2010). Therefore, it is essential to determine whether APC proteins have endogenous roles in high-fidelity chromosome segregation in vivo, and if so, by what mechanism they function.

To address these issues, we examined APC function in early Drosophila embryos, an excellent model for mitosis and cytoskeletal regulation. Early embryos are syncytial, with nuclei undergoing 13 synchronous mitotic cycles without cytokinesis (Mazumdar and Mazumdar, 2002). Cycles 10-13 occur cortically (Fig. 1A; supplementary material Movie 1), during which mitotic spindles form parallel to the cortex, so both daughter nuclei remain cortically anchored. Although there is no cytokinesis, actin-lined membrane invaginations called pseudocleavage furrows move down between nuclei at prophase (Fig. 1A, $\left.\mathrm{A}^{\prime}\right)$ and recede during anaphase. Pseudocleavage furrows separate adjacent nuclei/spindles during divisions, and are thought to provide attachment points for astral microtubules. Syncytial mitoses are extraordinarily rapid ( $\sim 10$ minutes) and thus more error-prone. Nuclei suffering mitotic defects are removed from the cortex so they do not contribute to the future embryo, a process termed 'nuclear fallout' (Sakurai et al., 2011; Takada et al., 2003). Thus, syncytial embryos are a powerful system in which to examine how cells achieve high-fidelity mitosis during normal development by investigating proteins with subtle yet important functions in mitotic fidelity.

Syncytial fly embryos are an excellent model for the cytoskeletal regulatory roles of APC in vivo. Embryos lacking APC2 have an elevated frequency of nuclear fallout, suggesting that APC2 promotes high-fidelity mitosis (McCartney et al., 2001; McCartney et al., 2006). Several mechanistic models have been suggested for how APC2 loss leads to elevated nuclear fallout. Because APC2 is cortically localized, it might tether mitotic spindles to the cortex (McCartney et al., 2001). Other work revealed potential roles for APC2 in building pseudocleavage furrows (Webb et al., 2009; Zhou et al., 2011). APC2 also helps promote centrosome separation (Buttrick et al., 2008; Cao et al., 2010), but this was not causally connected to nuclear removal. Thus, as in cultured cells, the mechanism by which APC2 regulates the cytoskeleton in vivo remains unclear.

To determine the mechanism by which APC2 helps prevent CIN, we identified the mitotic processes that do or do not require APC2. Together, our data reveal that APC2 acts as a cytoskeletal regulator to help maintain genome stability by promoting mitotic fidelity and preventing chromosome segregation errors. Our data further suggest the primary role of APC2 is in promoting centrosome separation, and this prevents formation of ectopic cleavage furrows. Interestingly, this requires almost all of the protein interaction domains of APC2, suggesting that a multiprotein complex operates at the cortex to ensure high-fidelity chromosome segregation and to prevent mitotic errors.

\section{MATERIALS AND METHODS}

\section{Genetics}

Mutations are described at http://flybase.org/ and supplementary material Table S1. Wild type (WT) was $y$ w. APC2 null embryos were progeny of $A P C 2^{g 10}$ females and males. Embryos were collected from apple juice-agar plates at 0-3 hours. Maternal $\mathrm{axin}^{\mathrm{S} 04423}$ null mutants were generated by the dominant female-sterile technique (Chou et al., 1993). We used the $c h k 2^{p 6}$ allele (Brodsky et al., 2004).

\section{Embryo immunofluorescence and live-Imaging}

Embryo immunofluorescence was performed as described in Roberts et al. (Roberts et al., 2011). Antibodies, probes and live-imaging markers are listed in supplementary material Table S1. Fixed images were acquired on Zeiss Pascal or 510 confocal microscopes. PhotoshopCS4 (Adobe, San Jose, CA, USA) was used to adjust levels so that the range of signals spanned the entire output grayscale and to adjust brightness and contrast. Live images were acquired on a Nikon TE2000-E microscope with Visitech InfinityHawk multi-point array scanner, using $40 \times, 60 \times$ or $100 \times$ Nikon objectives, a Ludl emission filter wheel with Semrock filters, and Hamamatsu ORCA R2 camera. Excitation was by $491 \mathrm{~nm}$ and $561 \mathrm{~nm}$ lasers. Movies were processed in ImageJ.

\section{Scoring ectopic cleavage furrows}

We live-imaged cycle 11-13 embryos expressing Moesin:GFP and His:RFP or mCherry: $\alpha$ Tub, with three to five $z$-slices per time point. We scored ectopic furrows as linear Moesin:GFP structures above nuclei that: bisected normal pseudocleavage furrows, were stable in positioning, and persisted $\geq 1$ minute. The number of ectopic furrows per total number of nuclei with normal furrows in the field of view, averaged across all embryos and cycles, was compared using Fisher's Exact Test (GraphPad). To examine relationships between centrosome position and ectopic furrows, we employed two strategies, combining WT and $A P C 2$ embryos. (1) We drew a line across the ectopic furrow during prophase/metaphase, and a line bisecting the centrosomes (Fig. 2D, upper inset), and used the Angle tool to calculate their acute angle in 32 nuclei with ectopic furrows from nine embryos, and compared these with a hypothetical random mean angle of $45^{\circ}$ using the one-sample $t$-test (GraphPad). (2) We measured the $x y$ distance between centrosome pairs associated with ectopic furrows $\left(\mathrm{d}_{\mathrm{E}}\right.$; Fig. 2D, lower inset), and measured the distances between centrosomes in four to six neighboring pairs lacking ectopic furrows $\left(\mathrm{d}_{\mathrm{N}}\right)$ and calculated their mean distance. To allow unbiased comparisons of data from different embryos, we standardized distances by dividing ectopic furrow distance by normal centrosome distance $\left(\mathrm{d}_{\mathrm{E}} / \mathrm{d}_{\mathrm{N}}\right)$, yielding a ratio of centrosome distance at ectopic furrows relative to those without ectopic furrows. These were compared using the Wilcoxon Matched Pairs test $(n=11$ embryos).

\section{RESULTS \\ APC2 promotes mitotic fidelity by ensuring correct chromosome segregation}

Some studies suggest that APC plays an essential mitotic role, regulating spindle assembly or the spindle assembly checkpoint (see Introduction). Drosophila APC2 allows us to test these hypotheses during normal development. Syncytial embryos maternally mutant for $A P C 2$ have significantly elevated nuclear fallout; fly APC1 (APC - FlyBase) does not play a role here (McCartney et al., 2001; McCartney et al., 2006). As some APC2 mutants encode dominantnegative proteins that might interfere with the cytoskeletal binding partners of APC2, we used embryos maternally and zygotically homozygous for the null allele $A P C 2^{g 10}$ (McCartney et al., 2006). Consistent with previous work, most divisions occurred successfully in $A P C 2^{g 10}$ mutants, but the percentage of nuclei undergoing fallout significantly increased (mean $=2.04 \%, n=51$ embryos; WT mean $=0.93 \%, n=60 ; P=0.0024$; Fig. $1 \mathrm{~A}-\mathrm{C}$ ). Thus, in syncytial embryos, APC proteins are not essential for mitosis, but instead ensure high fidelity.

To determine the root cause of elevated mitotic failure in $A P C 2$ mutants, we used live-imaging to compare mitosis in WT and $A P C 2$ embryos expressing the chromatin marker Histone2Av:RFP (His:RFP). We hypothesized that nuclear removal is a response to mitotic defects such as aberrant chromosome segregation. In WT and $A P C 2$ mutants, we found that nuclear fallout was frequently preceded by chromosome segregation defects, apparent as lagging chromosomes or chromosome bridges ( $88 \%$ had apparent defects, $n=23 / 26$ nuclei; Fig. 1D; supplementary material Fig. S1A and Movies 1, 2). The frequency of segregation defects was significantly elevated in APC2 embryos (2.1\%, $n=19 / 902$ dividing 

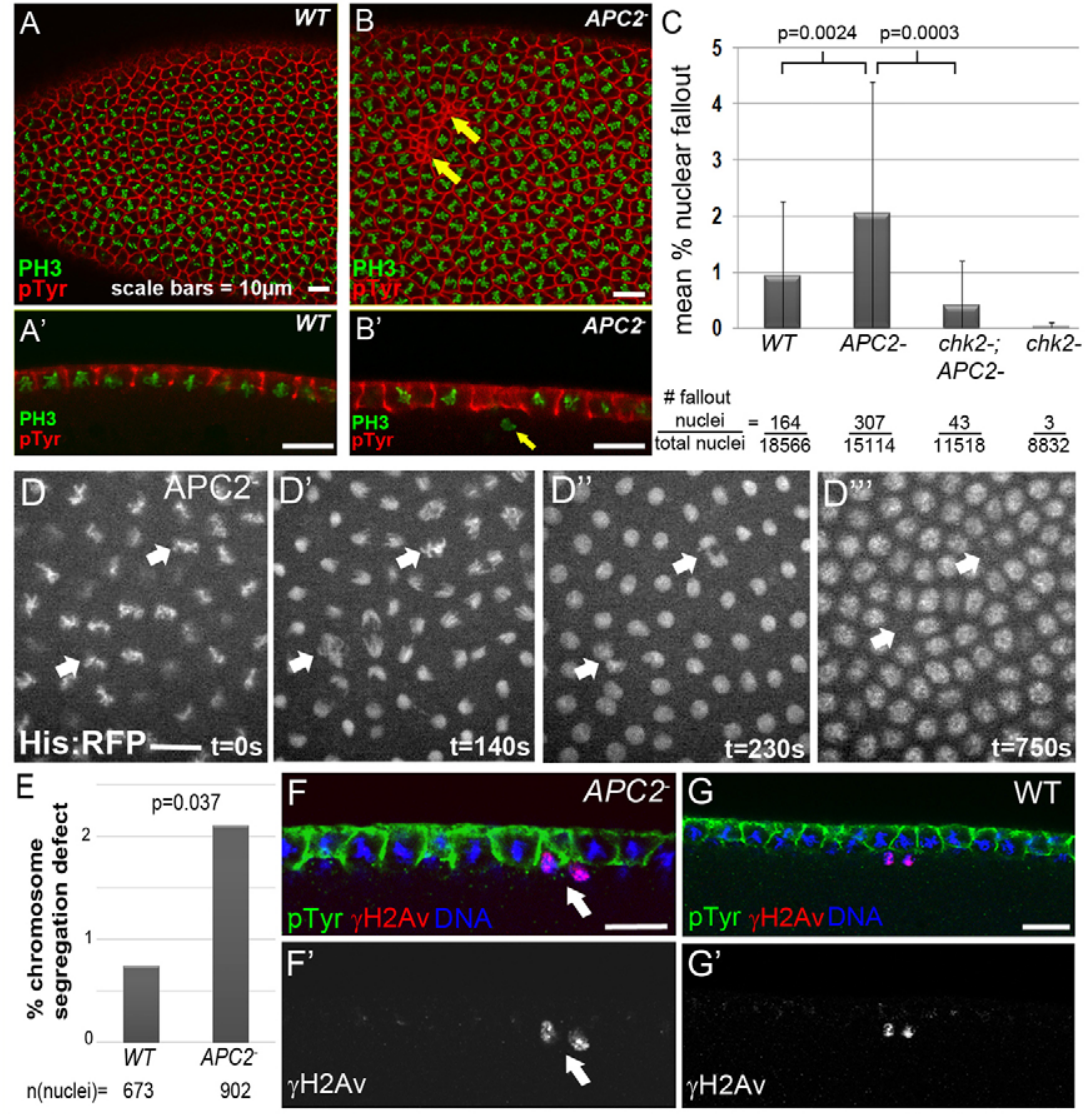
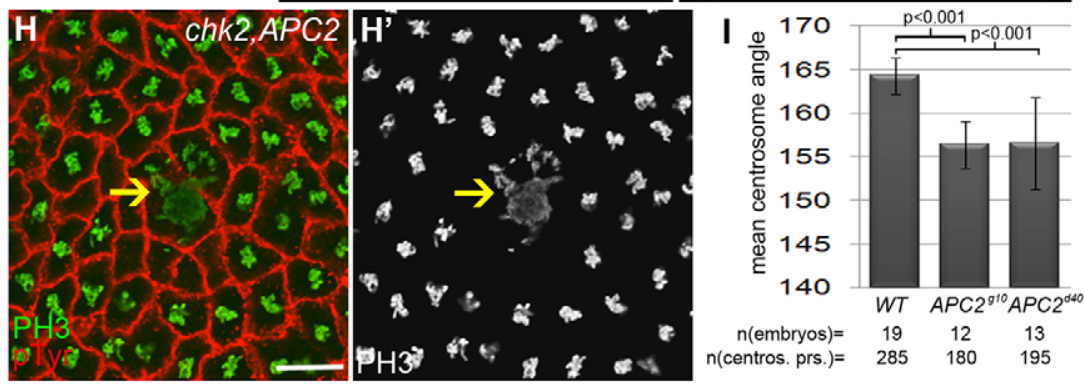

Fig. 1. APC2 helps prevent nuclear removal and promotes accurate chromosome segregation. (A-B') WT and APC2 Drosophila embryos labeled for mitotic nuclei ( $\mathrm{PH} 3$, phosphoHistone3; green) and pseudocleavage furrows (pTyr, phosphoTyrosine; red). $\left(A^{\prime}, B^{\prime}\right)$ Cross-sectional views of cortical nuclei and pseudocleavage furrows. Note region of nuclear removal ( $B$, arrows), and nucleus undergoing fallout ( $B^{\prime}$, arrow). (C) APC2 embryos had significantly more nuclear fallout than WT, and most fallout was inhibited in chk2;APC2 double mutants. (D-D'") APC2 embryo expressing the chromatin marker His:RFP. Chromosome mis-segregation ( $D^{\prime}, D^{\prime \prime}$, arrows) precedes nuclear removal ( $\mathrm{D}^{\prime \prime \prime}$, arrows). (E) APC2 embryos have significantly more chromosome segregation defects than do WT. (F-G') Nuclei undergoing fallout accumulate the DNA damage marker $\mathrm{YH} 2 \mathrm{Av}$ (red). $\left(\mathbf{H}, \mathbf{H}^{\prime}\right)$ In chk2;APC2 double mutants, nuclei are retained at the cortex and form fused aggregates (arrows). (I) Centrosome separation was significantly reduced in embryos mutant for APC2 ${ }^{170}$ (null allele) or APC2 ${ }^{d 40}$ (truncation allele) versus WT. Error bars represent s.d. The threshold $P<0.05$ was used in all tests; phenotype scoring and statistical tests are described in supplementary material Fig. S5. Scale bars: $10 \mu \mathrm{m}$. nuclei versus $0.7 \%, n=5 / 673$ in WT; $P=0.037$; Fig. $1 \mathrm{E})$. These data suggest that elevated rates of chromosome segregation defects are likely to be the cause of the increased nuclear fallout in $A P C 2$ embryos, consistent with the recent findings that embryos lacking the DNA helicase RecQ5 also have increased fallout following chromosome segregation errors (Sakurai et al., 2011). Furthermore, nuclear fallout can be initiated by a CHK2 (LOK FlyBase)-mediated response to induced DNA damage (Takada et al., 2003), and two recent studies found that chromosome segregation defects can generate such damage (Crasta et al., 2012; Janssen et al., 2011). In agreement, both $A P C 2$ mutant and WT nuclei undergoing fallout were highly enriched for the DNA damage marker $\gamma \mathrm{H} 2 \mathrm{Av}$ (HIS2AV - FlyBase) (Fig. 1F,G) (Madigan et al., 2002). To test the hypothesis that nuclear fallout in $A P C 2$ mutants was a response to DNA damage, we generated $c h k 2 ; A P C 2$ double-mutant embryos. Almost all fallout was inhibited in them; instead, defective nuclei remained at the cortex, forming fused aggregates (Fig. 1C,H). Together, these data suggest a model in which chromosome segregation defects generate DNA damage, activating the DNA damage response to initiate removal of defective nuclei.

\section{APC2 loss leads to centrosome separation defects and ectopic cleavage furrows}

To identify mechanisms by which APC2 promotes high-fidelity chromosome segregation, we examined underlying cytoskeletal events. Although some studies have suggested that APC proteins promote spindle assembly (Bahmanyar et al., 2009; Dikovskaya et al., 2004; Green et al., 2005), APC2 mutant spindles are structurally normal (McCartney et al., 2006). We therefore examined centrosome separation, as defects in this can trigger chromosome segregation errors (Silkworth et al., 2012; Tanenbaum and Medema, 2010). In syncytial embryos, centrosomes begin apically atop the nuclear envelope, and then move in both the $x y$ and $z$ axes; separating from one another in $\mathrm{S}$ phase, and away from the cortex during mitosis. $A P C 2^{\triangle S}$ mutants fail to fully separate centrosomes by prophase (Buttrick et al., 2008; Cao et al., 2010). However, $A P C 2^{\triangle S}$ is dominant-negative (McCartney et al., 2006). We therefore determined whether endogenous APC2 is required for centrosome separation. Indeed, $A P C 2$ null mutants had a significantly reduced mean centrosome separation angle at cycle $12 / 13$ prophase (WT mean $=164.6^{\circ}, n=240$ centrosome pairs, 16 embryos, versus $156.4^{\circ}$ in $A P C 2, n=180$ centrosome pairs, 12 
embryos; $P<0.001$; Fig. 1I). In $A P C 2$ null embryos, $12 \%$ of centrosome pairs had separation angles more than two standard deviations below the WT mean. Furthermore, WT centrosomes normally move away from the apical surface during mitosis (Cao et al., 2010), whereas in $A P C 2$ mutants centrosomes remained more apical $(2.15 \mu \mathrm{m}$ below apical surface versus $2.70 \mu \mathrm{m}$ in WT; $P=0.03$; supplementary material Fig. S1B-D). Thus, endogenous APC2 promotes proper centrosome separation.

In syncytial embryos, centrosomes have multiple roles. As in other cells, they nucleate spindle microtubules, but, as noted above, spindles are relatively normal in APC2 mutants. However, in syncytial embryos, centrosomes also regulate the actin cytoskeleton and thus formation/positioning of pseudocleavage furrows separating mitotic nuclei (Sullivan, 2009). We therefore examined whether chromosome segregation defects in APC2 mutants led to defects in pseudocleavage furrows, as others have suggested (Webb et al., 2009).

To test this hypothesis, we live-imaged $A P C 2$ mutants expressing His:RFP and the actin-binding protein Moesin:GFP (Moe:GFP), which marks pseudocleavage furrows. We did not observe substantial defects in positioning of pseudocleavage furrows between nuclei. Instead, live imaging revealed a novel cytoskeletal defect in APC2 mutants. During normal cytokinesis, cells assemble cleavage furrows where microtubules overlap, thus dividing the cell into two daughters. This is driven by centralspindlin complex recruitment and subsequent Rho activation (Barr and Gruneberg,
2007). However, syncytial embryos do not assemble cleavage furrows in the normal position; instead, they assemble pseudocleavage furrows between centrosomes of opposing nuclei (Fig. 2E). Recent work revealed that, during syncytial divisions, although centralspindlin proteins localize to the region of microtubule overlap, cleavage furrows do not form owing to failure to activate Rho. Misexpressing active Rho triggered formation of 'ectopic' furrows above nuclei, where cleavage furrows would form in normal cytokinesis (Crest et al., 2012). Strikingly, as APC2 mutants entered prophase, we observed similar ectopic furrows over a subset of nuclei (Fig. 2A-A"; supplementary material Movie 3). The ectopic furrows resembled cleavage and pseudocleavage furrows, similarly recruiting Moe:GFP, PavKLP:GFP and Diaphanous (DIA; supplementary material Fig. S1E,F) (Cao et al., 2008; Crest et al., 2012; Minestrini et al., 2003). Like pseudocleavage furrows, ectopic furrows were cell cycledependent; they formed at prophase and typically withdrew at anaphase (supplementary material Movie 3). Ectopic furrows were occasionally observed in WT, but were significantly less frequent than in $A P C 2$ mutants $(7.0 \%$ in $A P C 2$ versus $2.5 \%$ in WT; $P<0.0001$; Fig. 2C). Thus, APC2 reduces ectopic furrow frequency.

Because APC2 promotes centrosome separation, we hypothesized that ectopic furrows in $A P C 2$ mutants are caused by failure to fully separate centrosomes, allowing them to position an ectopic furrow between centrosomes above a nucleus, rather than positioning pseudocleavage furrows between centrosomes of
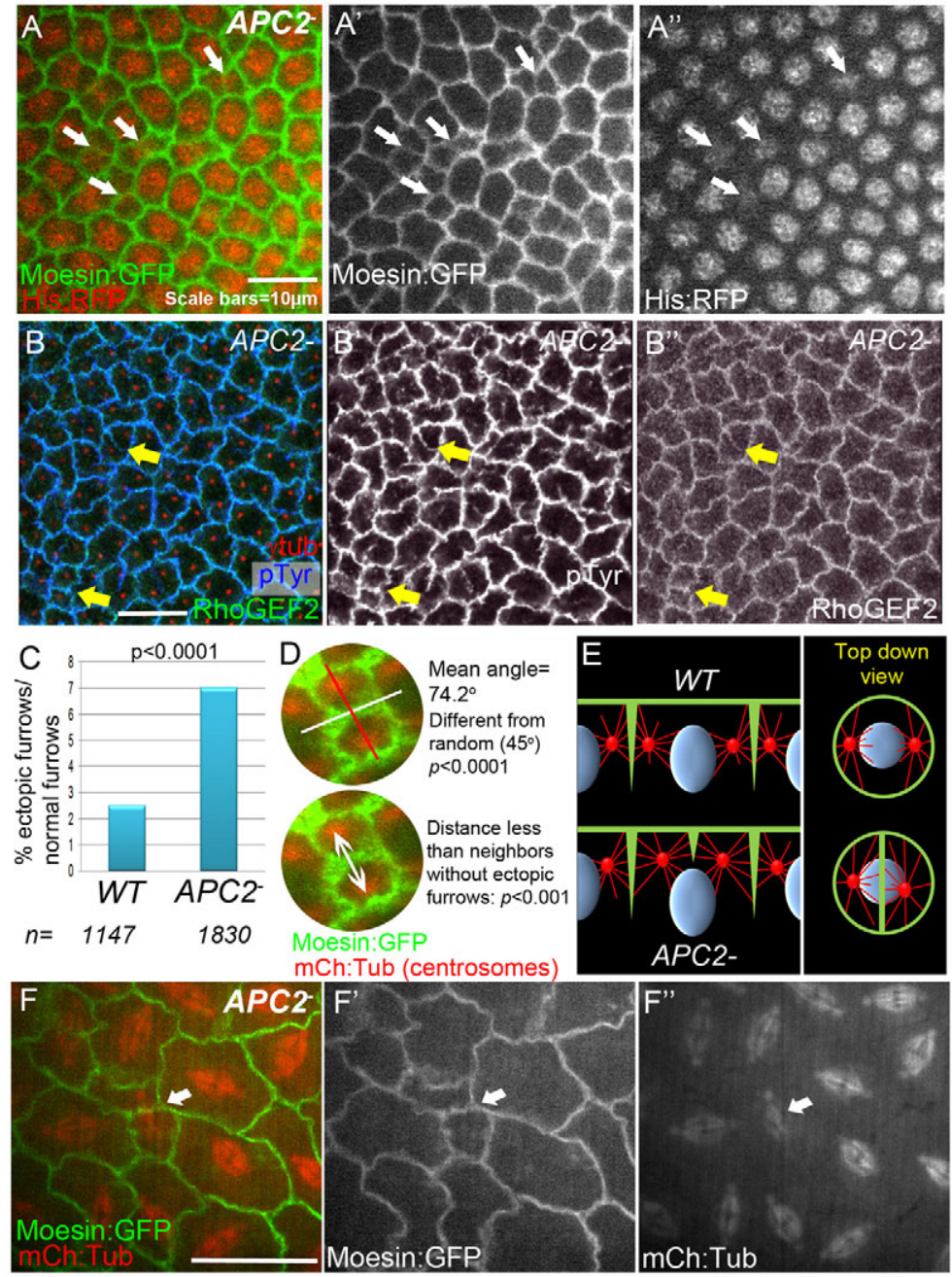

Fig. 2. Loss of APC2 elevates frequency of ectopic cleavage furrows that displace nuclei. $\left(\mathbf{A}-\mathrm{A}^{\prime \prime}\right)$ APC2 embryo. Moe:GFP (green) marks actin, His:RFP (red) marks nuclei. Note ectopic furrows over some nuclei ( $A^{\prime}$, arrows), which displace underlying nuclei away from cortex (A", arrows). (B-B") RhoGEF2 accumulates at ectopic furrows (arrows). (C) APC2 mutants have significantly more ectopic furrows than do WT. (D) Ectopic furrows (white line) form perpendicular to centrosome orientation (red line) (upper image), and centrosomes associated with ectopic furrows are closer together than centrosomes without ectopic furrows (lower image). Moe:GFP labels furrows and mCherry:aTubulin marks centrosomes. (E) Model depicting normal and ectopic furrow formation. In WT (top), centrosomes and microtubules (red) are fully separated allowing microtubule interactions between neighboring centrosomes to position pseudocleavage furrows (green) between nuclei (blue). In APC2 mutants (bottom), centrosomes do not fully separate, allowing microtubule interactions above nuclei, triggering ectopic furrows. (F-F") Ectopic furrows (arrows) can also disrupt positioning of underlying mitotic spindles (mCh:Tub, red). Scale bars: $10 \mu \mathrm{m}$. 
adjacent nuclei (Fig. 2E). Consistent with this, ectopic furrows bisected actin caps roughly perpendicular to centrosome orientation (mean angle $=74.2^{\circ}$; Fig. 2D, upper image). Strikingly, the distance between centrosomes associated with ectopic furrows (mean $=7.55 \pm 1.7 \mu \mathrm{m}$ ) was significantly less than that between centrosomes in neighboring cells without ectopic furrows (mean=8.0 $\pm 1.6 \mu \mathrm{m} ; P<0.001$; Fig. 2D, lower image). Together, these data and the known role of the centrosome in pseudocleavage furrows suggest that ectopic furrows result from centrosome separation defects. The ectopic furrows are similar to those induced by overexpressing the centrosome-associated protein Centrocortin (CEN) (Kao and Megraw, 2009). We thus tested the hypothesis that increased CEN levels were present at centrosomes associated with ectopic furrows. However, immunostaining did not reveal obvious CEN recruitment differences (data not shown). We next tested whether ectopic furrows might reflect inappropriate localization of Rho activators, as failure to recruit RhoGEF2 normally prevents ectopic furrow formation (Crest et al., 2012). Strikingly, ectopic furrows recruited RhoGEF2 (Fig. 2B), the absence of which normally prevents cleavage furrow assembly in syncytial divisions. Therefore, we favor a model in which, when centrosomes are too close together, inappropriate delivery of cues or cargo triggers furrow formation above nuclei rather than solely at the periphery (Fig. 2E).

\section{Ectopic furrows induced by APC2 loss can perturb nucleus and spindle positioning}

Because ectopic furrows formed directly above spindles, we hypothesized that the mitotic defects in $A P C 2$ mutants might be due to effects of ectopic furrows on spindles or associated nuclei. Consistent with this, ectopic furrows frequently displaced underlying nuclei away from the cortex $(60 \%, n=49 / 82$ ectopic furrows; Fig. 2A; supplementary material Movie 3). By live imaging, we observed that although many nuclei affected by ectopic furrows divided without incident, others had subsequent mitotic defects. In severe cases, the anaphase chromosome segregation plane was not parallel with the cortex (supplementary material Fig. S2 and Movie 4), and nuclei underwent fallout (supplementary material Movie 5). This suggested that mitotic spindles in affected nuclei might be perturbed. We thus imaged $A P C 2$ mutants expressing Moe:GFP and mCherry: $\alpha$ Tubulin (mCh:Tub). This revealed mis-positioned mitotic spindles associated with many ectopic furrows (34\%, $n=21 / 61$ ectopic furrows; Fig. 2F). Together, these data suggest that APC2 promotes high-fidelity chromosome segregation by facilitating centrosome separation, thus preventing ectopic furrows that can disrupt spindle organization and reduce chromosome segregation fidelity. Of course, APC2 loss might lead to chromosome mis-segregation by additional means; e.g. centrosome separation defects may disrupt chromosome segregation by increasing merotelic attachments (Silkworth et al., 2012).

\section{Perturbing centrosome separation by other means is associated with ectopic furrows and fallout}

To test further our model wherein incomplete centrosome separation leads to increased ectopic furrows, which in turn increases nuclear fallout, we sought to disrupt centrosome separation in an APC2independent manner. To do so, we expressed RNAi against the microtubule plus-tip protein EB1. EB1 regulates multiple aspects of microtubule function and disrupting EB1 function in syncytial embryos increases chromosome mis-segregation and nuclear fallout (Rogers et al., 2002; Webb et al., 2009). Importantly, unlike in mammals, APC2 lacks the C-terminal EB1-binding site, and EB1 does not interact genetically or physically with APC2 in syncytial embryos, suggesting that they function independently (Webb et al., 2009). Embryos expressing Ebl-RNAi had significantly reduced centrosome separation (mean $=156.6^{\circ} ; P=0.0004$ versus WT; Fig. 3A). To determine whether EB1 helped prevent ectopic furrows, we live-imaged Eb1-RNAi embryos expressing Moe:GFP and $\mathrm{mCh}$ :Tubulin. EB1 knockdown significantly increased ectopic furrow frequency $(P<0.0001$; Fig. 3B). As in $A P C 2$ mutants, ectopic furrows in Ebl-RNAi embryos sometimes disrupted underlying mitotic spindles (Fig. 3E). It is worth noting that the degree of centrosome separation defects and ectopic furrows after EB1 knockdown were both very similar to those in $A P C 2$ mutants, consistent with a direct relationship between reduced centrosome separation and ectopic furrows. Lastly, EB1 knockdown significantly increased nuclear fallout (mean=11.9\%, $P<0.0001$; Fig. 3C,D). Fallout rates after $E b 1$-RNAi were much greater than in $A P C 2$ mutants, probably reflecting the many roles of EB1 in microtubule regulation. Together, these experiments support our hypothesis that initial defects in centrosome separation help trigger ectopic furrows, which in some cases perturb mitosis, resulting in
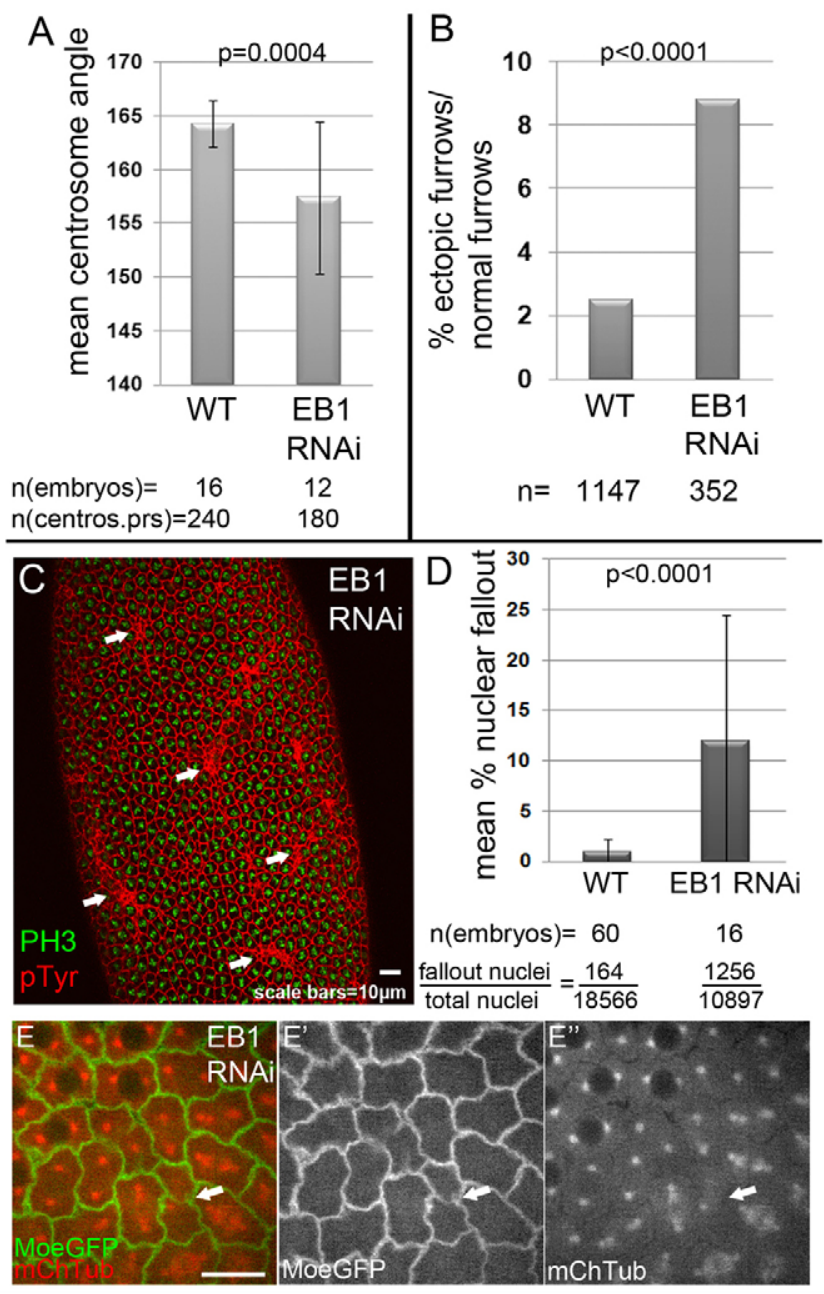

Fig. 3. EB1 knockdown leads to centrosome separation defects, ectopic furrows and nuclear fallout. (A) Maternally loaded RNAi against EB1 significantly reduced centrosome separation, relative to WT. (B) EB1 knockdown increased rates of ectopic furrows. (C,D) EB1 knockdown also led to increased nuclear fallout (arrows in C). (E-E") Some ectopic furrows displace the underlying mitotic spindle (arrows). Error bars represent s.d. Scale bars: $10 \mu \mathrm{m}$. 
nuclear fallout. This model is also consistent with the timing of these events; in $A P C 2$ mutants, incomplete centrosome separation results from slower separation throughout the previous $\mathrm{S}$ phase (Buttrick et al., 2008). Thus, incomplete centrosome separation temporally precedes ectopic furrows, chromosome mis-segregation, and, of course, nuclear removal. Like normal pseudocleavage furrows, ectopic furrows form at mitosis onset, mature at metaphase and retract at anaphase, and thus they form prior to chromosome segregation and nuclear fallout.

\section{APC2 functions at the embryonic cortex to ensure mitotic fidelity}

Centrosome separation is driven in part by forces generated by centrosome-nucleated microtubules, which interact with microtubules emanating from the other centrosome or with the cell cortex (Tanenbaum and Medema, 2010). APC can localize to mammalian centrosomes (Louie et al., 2004), but we have not observed APC2 at centrosomes in any normal fly tissue (Akong et al., 2002; McCartney et al., 1999). Instead, APC2 is predominantly cortical in early embryos (McCartney et al., 2001), suggesting that its role in promoting centrosome separation and preventing nuclear fallout occurs at the cortex. Consistent with this, $A P C 2$ alleles affecting its cortical localization also cause nuclear fallout (McCartney et al., 2006; Zhou et al., 2011). However, it remained possible that the dual effects on syncytial function and localization were simply a correlation rather than causal.

To test directly whether the cortical localization of APC2 is essential for its cytoskeletal functions, we generated a WT APC2 variant that could not localize cortically but instead was redirected to mitochondria. We then compared full-length APC2 $\left(\mathrm{APC} 2^{\mathrm{FL}}\right)$ with full-length $\mathrm{APC} 2$ with a $\mathrm{C}$-terminal mitochondriallocalization tag (APC2 ${ }^{\text {mito }}$ ), both expressed via the endogenous promoter, GFP-tagged, inserted into the same genomic location, and expressed at levels comparable to endogenous APC2 (Roberts et al., 2012). APC $2^{\text {mito }}$ re-localizes to mitochondria, but despite this, fully rescues Wnt signaling in both mammalian cells and flies (Roberts et al., 2012). We confirmed that these APC2 variants localized as expected in syncytial embryos, by driving their expression using the Gal4-UAS system. As expected, APC2 ${ }^{\mathrm{FL}}$ correctly localized to the cortex along pseudocleavage furrows (Fig. 4A), like endogenous APC2 (McCartney et al., 2001), whereas $\mathrm{APC} 2{ }^{\text {mito }}$ was completely mislocalized away from the cortex to internal structures, presumably mitochondria (Fig. 4B). This allowed us to test directly whether APC2 cortical localization is essential for cytoskeletal function.

We assessed this by expressing APC $2^{\text {mito }}$ in $A P C 2$ null embryos, and scoring nuclear fallout. $\mathrm{APC} 2{ }^{\mathrm{FL}}$ rescued nuclear fallout defects of $A P C 2$ embryos (mean $=1.12 \%, n=43 ; P=0.023$ versus $A P C 2$; not significantly different than WT; Fig. 4C). By contrast, APC $2{ }^{\text {mito }}$ did not reduce the fallout rate to WT levels (mean $=1.53 \%, n=39$; $P=0.038$ versus WT; not significantly different than $A P C 2$; Fig. $4 \mathrm{C}$ ). Together with earlier work on $A P C 2$ mis-sense mutants, this strongly suggests APC2 acts at the cortex to regulate high-fidelity mitosis, and underscores that this cytoskeletal role is Wnt independent.

\section{The cytoskeletal function of APC2 requires the Arm repeats and $\beta$ cat binding sites}

To determine the mechanism by which APC promotes genome stability, we also must identify proteins working with it in this process. Human and fly APCs all share multiple well-conserved protein interaction domains. All have N-terminal Armadillo (Arm) repeats binding multiple partners, including known cytoskeletal regulators. In addition, the C-terminal half of APC2 (equivalent to the 'mid' region of human APC) contains islands of protein conservation, defining known or putative protein-binding motifs, interspersed in regions of overall low conservation that are predicted to be unstructured. This region includes several 15- and 20-amino acid repeats $(15+20 \mathrm{aa})$ that each bind $\beta$ cat, and two SAMP motifs binding Axin, a scaffold protein important in Wnt signaling (Fig. 5A). These diverse partners allow for several models of the mechanisms of action of APC. By generating a set of mutants by precisely removing particular domains/motifs, we defined how different regions, and thus protein partners, contribute to the role of APC2 in Wnt regulation (Roberts et al., 2011). Here, we used these mutants to define the APC2 domains required to help ensure mitotic fidelity, and to identify putative partners in this process.

One model suggests that APC2 promotes interactions between astral microtubules and cortical actin through a complex including $\beta$ cat (supplementary material Fig. S4) (McCartney et al., 2001). Our new data suggest that APC2 facilitates centrosome separation, which promotes mitotic fidelity, probably by preventing ectopic furrow formation. This is consistent with a variant of our original model, in which cortical astral microtubule attachment facilitates centrosome separation. We thus investigated which protein interactions are essential for the mitotic stability role of APC2. The hypothesis that APC2 links to actin via $\alpha$ - and $\beta$ cat, and then interacts with astral microtubules via an unknown partner (APC2 lacks the direct microtubule-binding site of human APC), predicts that the ability of APC 2 to bind $\beta$ cat via its $15+20$ aa repeats would be crucial. It also predicts that the Arm repeats will be important, facilitating interaction with one of the microtubule-binding proteins interacting with them, e.g. Kinesin-associated protein 3 (KAP3) (Jimbo et al., 2002). By contrast, because the role of APC2 in Wnt signaling is not important in syncytial embryos (McCartney et al., 2006) (this study), we predicted that the ability of APC2 to bind to Axin via the SAMPs and thus assemble the Wnt-regulatory destruction complex would be dispensable.
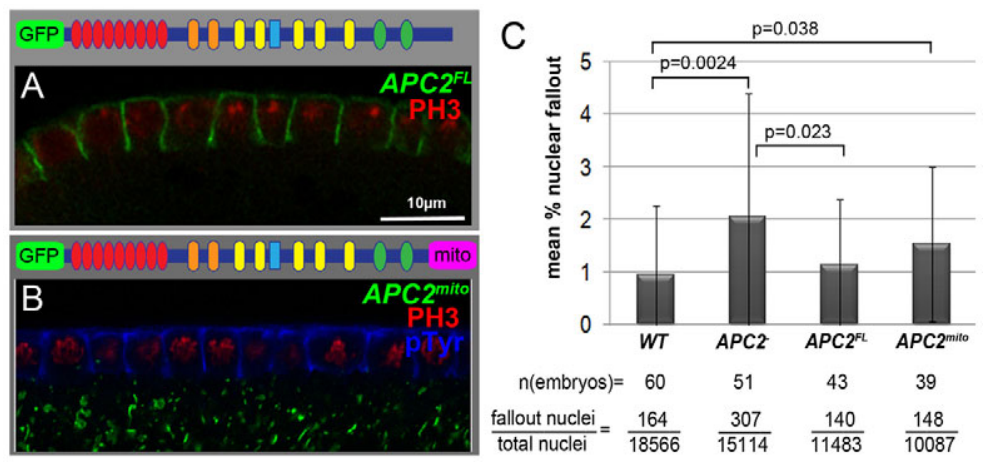

Fig. 4. APC2 functions at the embryonic cortex. (A) Fulllength GFP:APC2 (APC2 ${ }^{\mathrm{FL}}$, green) localizes to pseudocleavage furrows. (B) APC2 with a mitochondrial localization sequence (APC2 ${ }^{\text {mito }}$, green) is mislocalized away from pseudocleavage furrows (labeled with pTyr, blue). (C) APC2 ${ }^{\mathrm{FL}}$ in the APC2 null background restores nuclear fallout rate to WT levels, but fallout levels in APC2 null embryos expressing APC2 ${ }^{\text {mito }}$ remain significantly greater than WT, but are not significantly different from $A P C 2$ mutants. Comparisons not specifically indicated were non-significant $(P>0.05)$. Error bars represent s.d. 


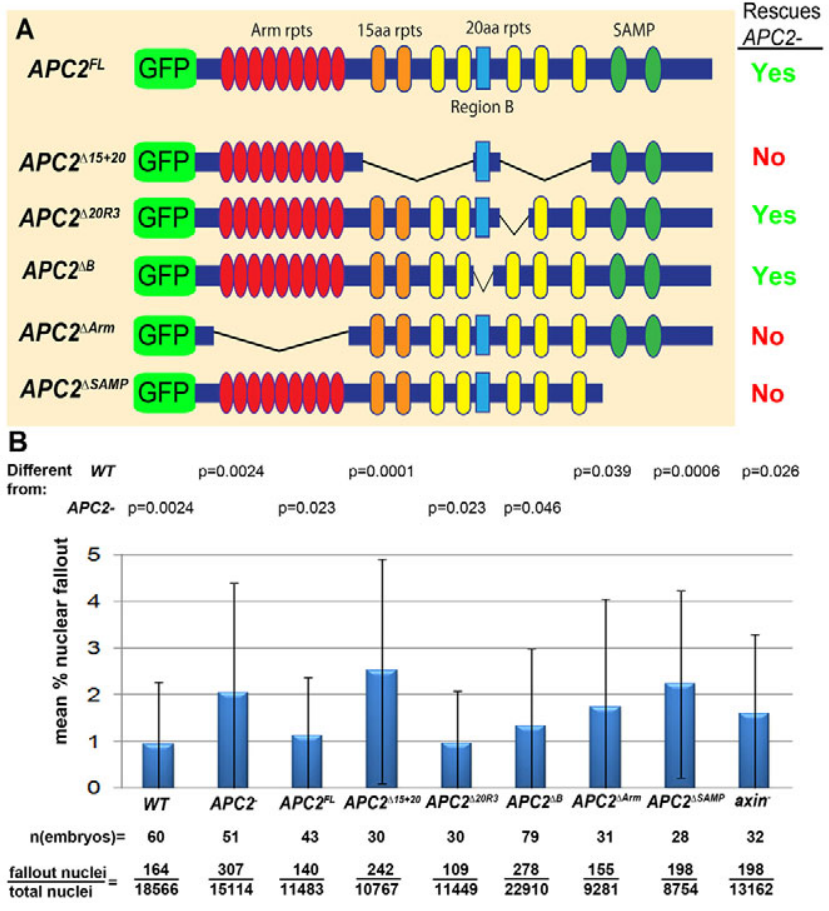

Fig. 5. The cytoskeletal function of $A P C 2$ requires multiple protein interactions, and Axin promotes mitotic fidelity. (A) Diagram of $A P C 2$ transgenes depicting full-length $\mathrm{APC2}\left(A P C^{F L}\right)$ and mutants with deletions of protein interaction domains. On the right is a summary of the data from $B$, indicating whether particular transgenes restored nuclear fallout rate to WT levels. (B) APC2 full-length rescued the null phenotype, as did transgenes lacking conserved region B or 20R3. However, transgenes lacking the Arm repeats, all 15+20aa repeats, or the SAMPs did not restore nuclear fallout levels to WT. Axin mutants also possessed significantly increased nuclear fallout relative to WT; this was not significantly different from APC2 embryos. Statistical comparisons not indicated were nonsignificant $(P>0.05)$. Error bars represent s.d.

To test these hypotheses directly and clarify mechanisms by which APC2 regulates the cytoskeleton, we determined which APC2 protein-interaction domains are necessary. To do so, we used a set of $A P C 2$ mutants in which different protein-binding regions had been deleted (Fig. 5A), expressed using the endogenous promoter and accumulating at levels comparable to endogenous APC2 (Roberts et al., 2011). The 15+20aa repeats each bind $\beta$ cat with different affinities (Liu et al., 2006). To test whether the ability of APC2 to bind $\beta$ cat is essential for its mechanism of action, we first tested an APC2 mutant with a clean deletion of all 15+20aa repeats $\left(\mathrm{APC} 2^{\triangle 15+20 \mathrm{aa}}\right)$. APC2 $2^{\Delta 15+20 \mathrm{aa}}$ expressed in the null mutant background did not rescue nuclear fallout (mean $=2.50 \%, n=30$; $P=0.0001$ versus WT; Fig. 5B), supporting the hypothesis that APC2 regulates the cytoskeleton together with $\beta$ cat (McCartney et al., 2001). To test this hypothesis further, we used RNAi to knock down $\beta$ cat (Armadillo in Drosophila). Similar to APC2 mutants, $\beta$ cat-RNAi significantly decreased centrosome separation (mean $=161.1^{\circ} ; P<0.003$ versus WT; supplementary material Fig. S3A), and increased ectopic cleavage furrow frequency (mean=7.1\%; $P<0.0001$ versus WT; supplementary material Fig. S3B) and nuclear fallout (mean $=1.66 \% ; P=0.03$ versus WT; supplementary material Fig. S3C,D).

We next examined whether the cytoskeletal function of APC2 requires all the $15+20$ aa repeats. The highest-affinity $\beta$ cat-binding site is 20aa repeat3 (Liu et al., 2006), and this site was predicted to have special roles in Wnt regulation. Interestingly, removing 20aa repeat 3 did not disrupt the ability of $\mathrm{APC} 2^{\triangle 20 \mathrm{R} 3}$ to restore mitotic fidelity (mean $=0.95 \%, n=30 ; P=0.023$ versus $A P C 2$ null; Fig. 5B). Thus, our data support a model in which APC2 and $\beta$ cat work together in cytoskeletal regulation, and suggest that the $\beta$ cat-binding sites are collectively, but not individually, essential.

The Arm repeats of APC bind many partners, including KAP3, IQGAP, ASEF (ARHGEF4), CDC42 and PP2A (Jimbo et al., 2002; Kawasaki et al., 2000; Seeling et al., 1999; Sudhaharan et al., 2011; Watanabe et al., 2004). Mis-sense mutations in the Arm repeats disrupt the ability of APC2 to prevent nuclear fallout, but at least one has a stronger phenotype than the null mutant (McCartney et al., 2006), suggesting that these mutants might non-productively interact with APC2 partners, causing defects by sequestering partners rather than because the Arm repeats are essential. To assess cleanly the importance of the Arm repeats in cytoskeletal function, we tested an $A P C 2$ mutant lacking all the Arm repeats $\left(A P C 2^{\triangle A r m}\right)$. $\mathrm{APC} 2^{\triangle \mathrm{Arm}}$ did not significantly reduce fallout (mean $=1.73 \%, n=31$; $P=0.039$ versus WT; Fig. 5B), demonstrating that the Arm repeats are required for the cytoskeletal role of APC2. Further work is required to identify the Arm repeat-binding partners working with APC2 in syncytial embryos.

Recently, we identified a novel role for another APC2 motif, conserved region B, in Wnt regulation (Roberts et al., 2011). The mechanistic function of Region B is unknown, but we hypothesize that it is a protein interaction motif the binding partner(s) of which regulate the catalytic cycle of the $\beta$ cat destruction complex. To determine whether region $\mathrm{B}$ is essential for the cytoskeletal function of $\mathrm{APC} 2$, we assayed whether a mutant lacking it $\left(A P C 2^{\triangle B}\right)$ rescued fallout. Although $\mathrm{APC} 2^{\triangle \mathrm{B}}$ is non-functional in Wnt signaling (Roberts et al., 2011), APC $2^{\triangle \mathrm{B}}$ significantly reduces nuclear fallout (statistically indistinguishable from WT and significantly different from $A P C 2$; mean $=1.33 \%, n=79 ; P=0.046$; Fig. 5B). Thus, although region $\mathrm{B}$ is crucial for Wnt signaling, it appears to be dispensable for ensuring mitotic fidelity, supporting the idea that the dual roles of APC2 in cytoskeletal and Wnt regulation are mechanistically distinct.

\section{APC2 binding to Axin is important for cytoskeletal regulation and preventing nuclear fallout}

The SAMP repeats of APC2 bind Axin, the scaffold of the Wntregulatory destruction complex. Syncytial embryos lack Wnt signaling, and thus we hypothesized that the SAMPs would be dispensable for the activity of APC2. Surprisingly, APC2 $2^{\triangle \mathrm{SAMP}} \mathrm{did}$ not rescue nuclear fallout (Fig. 5B; mean $=2.26 \%, n=28 ; P=0.0006$ versus WT), suggesting that Axin might also regulate the cytoskeleton. To test this directly, we generated embryos maternally mutant for the null axin $^{\text {S04423 }}$ allele (Hamada et al., 1999). Like APC2 embryos, Axin mutants had elevated nuclear fallout (mean $=1.62 \%, n=37$; significantly greater than WT, $P=0.026$; not significantly different from $A P C 2$; Fig. 5B). Thus, Axin and its interaction with APC2 are both important for mitotic fidelity, consistent with the hypothesis that they work together in this process.

It remained possible that Axin promotes nuclear fidelity by a distinct, APC-independent mechanism. Our data suggest that APC2 facilitates high-fidelity mitosis by promoting centrosome separation and preventing ectopic furrows. To determine whether Axin operates by similar or distinct mechanisms, we analyzed these events. Axin mutants had reduced centrosome separation (Fig. 6; 


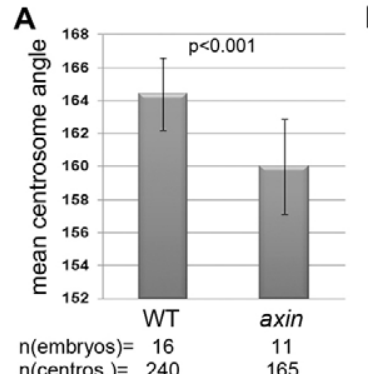

B
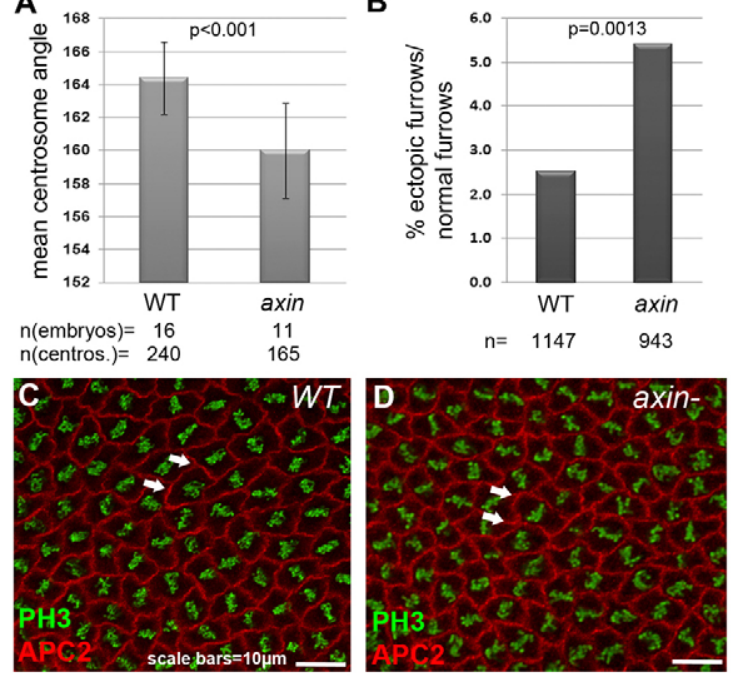

Fig. 6. Axin promotes centrosome separation and prevents ectopic furrows. (A) Centrosome separation was significantly reduced in Axin mutants. (B) Axin mutants possessed significantly more ectopic cleavage furrows. (C,D) Localization of endogenous APC2 to pseudocleavage furrows (arrows) appeared to be unaffected in Axin mutants. Error bars represent s.d. Scale bars: $10 \mu \mathrm{m}$.

mean $=160.0^{\circ}, n=180$ centrosome pairs; 12 embryos; significantly less than WT, $P<0.001$; not significantly different from $A P C 2$ ), and significantly more ectopic furrows than WT (Fig. 6; 5.4\%; $P=0.0013$ versus WT; not significantly different from $A P C 2)$. These data support the hypothesis that APC2 and Axin work in concert to ensure mitotic fidelity.

We next explored what role Axin plays in the cytoskeletal regulatory role of APC2. APC2 cortical localization is essential for cytoskeletal function, and this requires the action of another destruction complex protein, GSK3 (SGG - FlyBase) (McCartney et al., 2001). Because Axin is the scaffold in the destruction complex, we tested the hypothesis that Axin regulates APC2 cortical localization, perhaps by templating GSK3 phosphorylation of APC2 or $\beta$ cat. However, in contrast to GSK3 (McCartney et al., 2001), Axin was not required for cortical localization of APC2 (Fig. 6C,D). Together, these data support a model in which APC2 and Axin work together in syncytial embryos, identifying Axin as a novel cytoskeletal regulator in this system.

\section{DISCUSSION}

APC plays a key role in colon cancer initiation. Because of its known cytoskeletal binding partners and roles, it has been suggested that $A P C$ mutation also promotes cancer progression through increased CIN. However, despite numerous studies on APC function in cultured cells, there is no consensus as to the mechanisms by which it promotes mitotic fidelity (Rusan and Peifer, 2008), or about how important this is in vivo in whole animals.

\section{APC promotes high-fidelity mitosis through its role as a cytoskeletal regulator}

We used fly embryos to explore how APC proteins regulate mitotic fidelity in vivo. Our findings corroborate studies in mammalian cells suggesting that APC promotes genomic stability through its cytoskeletal functions (reviewed by Rusan and Peifer, 2008). Mammalian APC may also promote genomic stability by regulating Wnt signaling (Aoki et al., 2007; Hadjihannas et al., 2006), but our data indicate that the role of APC2 in preventing CIN in syncytial embryos does not involve this. Furthermore, although $A P C 2$ mutants have increased mitotic defects, most mitoses proceed without error. Thus, APC2 is not a central part of the mitotic apparatus in vivo, which contrasts with studies suggesting that APC proteins are key mitotic regulators. Instead, our data suggest that APC2 ensures high-fidelity mitosis. Although this might reflect functional differences between human and fly APCs, it may also suggest that cultured cells represent a sensitized situation with an elevated mitotic error rate, in which removal of fidelity regulators such as APC has a greater impact; consistent with this, cultured cells are prone to karyotypic anomalies (Drexler et al., 2000; Liu et al., 1997; Longo et al., 1997). It is important to note that the precise series of events triggered by APC2 loss in syncytial fly embryos are likely to be specific to that system, with APC playing diverse cytoskeletal roles at different times and places, both within the same animal and between species.

\section{Centrosome separation, ectopic furrows and DNA damage}

The small but significant increase in mitotic failure in $A P C 2$ mutants prompted us to work backwards from nuclear removal, reasoning that the primary defect might be significantly more frequent, but might not always trigger nuclear removal. Mechanistically, our data are consistent with a model wherein APC2 promotes high-fidelity chromosome segregation through a primary role in facilitating centrosome separation. In $A P C 2$ mutants, centrosome separation defects are highly elevated, with $12 \%$ of nuclei having separation reduced more than two standard deviations from the WT mean. Although this did not have apparent effects on spindle structure (McCartney et al., 2006), it had an unexpected consequence. APC2 mutants had a dramatic increase in ectopic cleavage furrows, the likelihood of which was highly correlated with reduced centrosome separation. The possible causal connection between incomplete centrosome separation and ectopic furrowing was further supported by our analysis of EB1 knockdown, as EB1 acts independently of APC2 in syncytial embryos (Webb et al., 2009). Although we contend that the similar effects of Eb1-RNAi and APC2 loss on centrosome separation and ectopic furrowing support the role of these phenotypes in downstream chromosome mis-segregation and nuclear fallout, it should be noted that EB1 appears to play a direct role in chromosome segregation. Thus, it is possible that this shared defect in chromosome segregation is responsible for nuclear fallout in both mutants, irrespective of their similarities in centrosome separation defects and ectopic furrows.

Ectopic furrows form where cleavage furrows would form in standard mitotic divisions. Indeed, formation of normal cytokinetic furrows is prevented in syncytial divisions by preventing Rho activation in the spindle midzone; although centralspindlin proteins localize there, RhoGEF2 does not, and artificial elevation of Rho activity triggers ectopic furrows (Crest et al., 2012). Our data suggest how ectopic furrows are normally prevented in early embryos. They are consistent with the hypothesis that proper centrosome separation leads to furrow formation around the dividing 'cell', rather than over the metaphase plate where it would form in normal cytokinesis (Fig. 2E). When centrosome separation is reduced, the ectopic RhoGEF2 recruitment we observed at the spindle midzone might be sufficient to trigger ectopic furrowing. Our model suggests that failure to fully separate centrosomes and subsequent formation of ectopic furrows then leads to mitotic defects. We found that nuclei and associated mitotic spindles can be physically displaced by 
ingressing ectopic furrows, perhaps disrupting chromosome segregation. This provides strong selective pressure for the existing mechanism preventing ectopic furrowing.

How then does this trigger nuclear removal? Nuclear removal can be initiated by DNA damage, monitored by the DNA damage sensor CHK2 (Antoni et al., 2007; Takada et al., 2003). Strikingly, APC2 mutants had more chromosome segregation errors than wild type, many of which were followed by nuclear removal. Furthermore, nuclei undergoing fallout accumulated the DNA damage marker $\gamma \mathrm{H} 2 \mathrm{Av}$. These data are consistent with the hypothesis that in $A P C 2$ mutants, chromosome mis-segregation generates DNA damage, thus triggering CHK2-mediated nuclear removal. Indeed, nuclear removal was blocked in $c h k 2 ; A P C 2$ double mutants. Recent studies in mammalian cells indicate that chromosome mis-segregation can cause DNA damage (Crasta et al., 2012; Janssen et al., 2011); intriguingly, one suggests cytokinetic furrow ingression on lagging chromosomes damages DNA (Janssen et al., 2011). Given the significant increase in lagging chromosomes and the ectopic cleavage furrows over spindles in $A P C 2$ mutants, it is tempting to speculate that a similar mechanism for generating DNA damage might be involved.

Of course, APC2 loss also might lead to chromosome segregation errors by other mechanisms. For example, centrosome separation defects are sufficient to generate merotelic microtubule attachments, thus leading to chromosome mis-segregation (Silkworth et al., 2012). The rapidity of syncytial divisions may make early embryos particularly sensitive to this type of error. Furthermore, in syncytial embryos the shared cytoplasm might make it difficult to alter timing of the metaphase-anaphase transition locally at an affected nucleus to allow repair of microtubule attachment errors. Together, our data suggest that although APC2 loss may lead to chromosome missegregation and nuclear fallout by multiple means, most if not all of these pathways begin with defects in centrosome separation. We believe our findings on the role of APC2 in mitotic fidelity in syncytial embryos may extend to other cell types, as fly neural stem cells lacking APC proteins have a significantly longer mitotic cycle, suggesting mitotic defects (Rusan et al., 2008). It will be interesting to see whether human APC promotes mitotic fidelity, at least in part, by regulating centrosome positioning; consistent with this, human APC facilitates centrosome movement in migrating neurons by stabilizing microtubule interactions with cortical actin (Asada and Sanada, 2010). Furthermore, in human colon cancer cells, merlin (neurofibromin 2), ezrin and APC2 govern centrosome and spindle positioning by regulating astral microtubule attachment to cortical actin (Hebert et al., 2012), suggesting a conserved role for APC in positioning centrosomes and spindles. Our findings may also help us to understand better the role of APC mutations in cancer. In most colon cancers, one $A P C$ allele bears a premature stop codon in the mid-region, truncating the protein. A fly mutant $\left(A P C 2^{d 40}\right)$ that mimics these truncations has increased nuclear fallout, like $A P C 2$ null embryos (McCartney et al., 2006). We found $A P C 2^{d 40}$ mutants also have reduced centrosome separation (Fig. 1I; mean $=156.6^{\circ}$; significantly different from WT; $P<0.001$, not significantly different from $A P C 2^{g 10}$ ). This suggests that at least some aspects of our model describing the mechanistic role of APC2 in promoting mitotic fidelity may apply to colon tumors with truncated APC.

Our data also test key aspects of models describing where and how APC2 regulates the cytoskeleton. Our analyses suggest that APC2 acts at the embryonic cortex, where it binds $\beta$ cat. These findings, together with the role of APC2 in centrosome separation, led us to revise our previous model (McCartney et al., 2001). We propose that APC2 facilitates stable interaction of astral microtubules with cortical actin to promote centrosome separation (supplementary material Fig. S4, left). This model is consistent with numerous studies indicating that cortical microtubule attachment helps mediate centrosome separation by generating pulling forces on astral microtubules through cortical dynein (Tanenbaum and Medema, 2010). It is now important to explore the effects of APC2 on microtubule dynamics directly.

\section{Axin and cytoskeletal regulation}

Our analyses of APC2 domain-deleted mutants revealed two additional mechanistic insights. First, the Arm repeats of APC2 are necessary for syncytial cytoskeletal function, suggesting that they bind an important partner. Based on our model that APC2 facilitates microtubule-cortex interactions, one attractive candidate is KAP3 (Sarpal et al., 2003). Second, the SAMP repeats, which allow APC2 to bind the destruction complex scaffold Axin, are also required for the cytoskeletal role of APC2. Furthermore, Axin mutants, like $A P C 2$ mutants, had reduced centrosome separation, and increased ectopic furrows and nuclear fallout. These data suggest that in syncytial embryos, APC2 acts as part of a multiprotein complex sharing many components with the Wnt-regulatory destruction complex. However, comparing APC2 mutants rescuing mitotic fidelity (this study) or Wnt signaling (Roberts et al., 2011; Roberts et al., 2012) strongly suggests that in syncytial embryos this complex has a distinct role from its Wnt-regulatory function. It will be important to determine the role of Axin in this complex, as we found it is not required to localize APC2 to the cortex, unlike the destruction complex component GSK3 (McCartney et al., 2001).

\section{Mitotic fidelity and CIN}

Our in vivo studies indicate that APC helps to ensure mitotic fidelity via cytoskeletal regulation, but show that, at least in syncytial fly embryos, this contribution is relatively subtle. Furthermore, our data suggest that embryos possess mechanisms to help compensate for defects caused by APC2 loss: although centrosomal separation defects affect $12 \%$ of nuclei, only $7 \%$ have ectopic furrows and only $2 \%$ experience chromosome segregation defects or nuclear fallout (supplementary material Fig. S4, right). It will be important to identify mechanisms buffering the effects of APC2 loss and thus reducing mitotic defects.

Although the precise role that APC2 plays in syncytial embryos and the cascade of consequences of APC2 loss are likely to be confined to that system, we hypothesize that APC proteins play analogous roles in other tissues, subtly regulating the cytoskeleton to promote mitotic fidelity. This is consistent with observations in cultured mammalian cells, which suggest that APC loss exerts diverse effects on the cytoskeleton, reducing mitotic fidelity (e.g. Draviam et al., 2006; Dikovskaya et al., 2007; Green and Kaplan, 2003). Such roles for APC proteins in mitotic fidelity and the possible mechanisms compensating for APC loss may have interesting implications for cancer progression. In colon polyps and early adenomas, prior to accumulation of additional mutations present in carcinomas, cells prone to massive mitotic defects would probably be eliminated by surveillance processes, such as DNA damage checkpoints, whereas cells with subtle perturbations of mitotic fidelity like those caused by APC2 loss might persist. Occasional mitotic errors may then help induce genomic instability spurring tumor progression. Furthermore, APC mutation might sensitize cells to checkpoint loss, as cytoskeletal defects caused by APC loss may be less effectively buffered, thereby elevating CIN. This provides a clear, testable model for how APC mutation contributes to tumor initiation and progression. 


\section{Acknowledgements}

We thank J. Sekelsky, V. Bautch, B. Duronio and N. Rusan for comments; D. Kiehart, S. Wasserman, N. Rusan, S. Rogers, K. McKim, W. Theurkauf and D. Glover for sharing reagents; and C. Allred and L. Kotlyanskaya for technical assistance.

\section{Funding}

This work was supported by the National Institutes of Health (NIH) [R01 GM67236 to M.P.; T32 CA 009156 and F32 GM095127 to J.S.P.; F32 GM076898 to D.M.R.]; start-up funds from Franklin and Marshall College [D.M.R.]; and a University of North Carolina Summer Undergraduate Research Fellowship (UNC SURF) [F.W.M.]. Deposited in PMC for release after 12 months.

\section{Competing interests statement}

The authors declare no competing financial interests.

\section{Author contributions}

J.S.P., F.W.M. and D.M.R. conducted experiments; J.S.P. and M.P. designed experiments; J.S.P. and M.P. prepared the manuscript.

\section{Supplementary material}

Supplementary material available online at

http://dev.biologists.org/lookup/suppl/doi:10.1242/dev.094425/-/DC1

\section{References}

Akong, K., McCartney, B. M. and Peifer, M. (2002). Drosophila APC2 and APC1 have overlapping roles in the larval brain despite their distinct intracellular localizations. Dev. Biol. 250, 71-90.

Alberici, P. and Fodde, R. (2006). The role of the APC tumor suppressor in chromosomal instability. Genome Dyn. 1, 149-170.

Antoni, L., Sodha, N., Collins, I. and Garrett, M. D. (2007). CHK2 kinase: cancer susceptibility and cancer therapy - two sides of the same coin? Nat. Rev. Cancer 7, 925-936.

Aoki, K., Aoki, M., Sugai, M., Harada, N., Miyoshi, H., Tsukamoto, T. Mizoshita, T., Tatematsu, M., Seno, H., Chiba, T. et al. (2007). Chromosomal instability by beta-catenin/TCF transcription in APC or beta-catenin mutant cells. Oncogene 26, 3511-3520.

Asada, N. and Sanada, K. (2010). LKB1-mediated spatial control of GSK3beta and adenomatous polyposis coli contributes to centrosomal forward movement and neuronal migration in the developing neocortex. J. Neurosci. 30, 8852-8865.

Bahmanyar, S., Nelson, W. J. and Barth, A. I. (2009). Role of APC and its binding partners in regulating microtubules in mitosis. Adv. Exp. Med. Biol. 656 $65-74$.

Barr, F. A. and Gruneberg, U. (2007). Cytokinesis: placing and making the final cut. Cell 131, 847-860.

Brodsky, M. H., Weinert, B. T., Tsang, G., Rong, Y. S., McGinnis, N. M., Golic, K. G., Rio, D. C. and Rubin, G. M. (2004). Drosophila melanogaster MNK/Chk2 and p53 regulate multiple DNA repair and apoptotic pathways following DNA damage. Mol. Cell. Biol. 24, 1219-1231.

Buttrick, G. J., Beaumont, L. M., Leitch, J., Yau, C., Hughes, J. R. and

Wakefield, J. G. (2008). Akt regulates centrosome migration and spindle orientation in the early Drosophila melanogaster embryo. J. Cell Biol. 180, 537 548.

Caldwell, C. M., Green, R. A. and Kaplan, K. B. (2007). APC mutations lead to cytokinetic failures in vitro and tetraploid genotypes in Min mice. J. Cell Biol. 178, 1109-1120.

Cao, J., Albertson, R., Riggs, B., Field, C. M. and Sullivan, W. (2008). Nuf, a Rab11 effector, maintains cytokinetic furrow integrity by promoting local actin polymerization. J. Cell Biol. 182, 301-313.

Cao, J., Crest, J., Fasulo, B. and Sullivan, W. (2010). Cortical actin dynamics facilitate early-stage centrosome separation. Curr. Biol. 20, 770-776.

Cardoso, J., Molenaar, L., de Menezes, R. X., van Leerdam, M., Rosenberg, C., Möslein, G., Sampson, J., Morreau, H., Boer, J. M. and Fodde, R. (2006) Chromosomal instability in MYH- and APC-mutant adenomatous polyps. Cancer Res. 66, 2514-2519.

Cheung, A. F., Carter, A. M., Kostova, K. K., Woodruff, J. F., Crowley, D., Bronson, R. T., Haigis, K. M. and Jacks, T. (2010). Complete deletion of Apc results in severe polyposis in mice. Oncogene $\mathbf{2 9}, 1857-1864$.

Chou, T. B., Noll, E. and Perrimon, N. (1993). Autosomal P[ovoD1] dominant female-sterile insertions in Drosophila and their use in generating germ-line chimeras. Development 119, 1359-1369.

Cimini, D., Tanzarella, C. and Degrassi, F. (1999). Differences in malsegregation rates obtained by scoring ana-telophases or binucleate cells. Mutagenesis $\mathbf{1 4}$ 563-568

Crasta, K., Ganem, N. J., Dagher, R., Lantermann, A. B., Ivanova, E. V., Pan, Y., Nezi, L., Protopopov, A., Chowdhury, D. and Pellman, D. (2012). DNA breaks and chromosome pulverization from errors in mitosis. Nature $\mathbf{4 8 2}, 53$ 58

Crest, J., Concha-Moore, K. and Sullivan, W. (2012). RhoGEF and positioning of rappaport-like furrows in the early Drosophila embryo. Curr. Biol. 22, 20372041.

Dikovskaya, D., Newton, I. P. and Näthke, I. S. (2004). The adenomatous polyposis coli protein is required for the formation of robust spindles formed in CSF Xenopus extracts. Mol. Biol. Cell 15, 2978-2991.

Dikovskaya, D., Schiffmann, D., Newton, I. P., Oakley, A., Kroboth, K., Sansom, O., Jamieson, T. J., Meniel, V., Clarke, A. and Näthke, I. S. (2007) Loss of APC induces polyploidy as a result of a combination of defects in mitosis and apoptosis. J. Cell Biol. 176, 183-195.

Draviam, V. M., Shapiro, I., Aldridge, B. and Sorger, P. K. (2006), Misorientation and reduced stretching of aligned sister kinetochores promote chromosome missegregation in EB1- or APC-depleted cells. EMBO J. 25, 28142827.

Drexler, H. G., Matsuo, A. Y. and MacLeod, R. A. (2000). Continuous hematopoietic cell lines as model systems for leukemia-lymphoma research. Leuk. Res. 24, 881-911.

Farrell, P. J., Allan, G. J., Shanahan, F., Vousden, K. H. and Crook, T. (1991). p53 is frequently mutated in Burkitt's lymphoma cell lines. EMBO J. 10, 28792887

Fearon, E. R. (2011). Molecular genetics of colorectal cancer. Annu. Rev. Pathol. 6, 479-507.

Fodde, R., Kuipers, J., Rosenberg, C., Smits, R., Kielman, M., Gaspar, C., van Es, J. H., Breukel, C., Wiegant, J., Giles, R. H. et al. (2001). Mutations in the APC tumour suppressor gene cause chromosomal instability. Nat. Cell Biol. 3 433-438

Gordon, D. J., Resio, B. and Pellman, D. (2012). Causes and consequences of aneuploidy in cancer. Nat. Rev. Genet. 13, 189-203.

Green, R. A. and Kaplan, K. B. (2003). Chromosome instability in colorectal tumor cells is associated with defects in microtubule plus-end attachments caused by a dominant mutation in APC. J. Cell Biol. 163, 949-961.

Green, R. A., Wollman, R. and Kaplan, K. B. (2005). APC and EB1 function together in mitosis to regulate spindle dynamics and chromosome alignment. Mol. Biol. Cell 16, 4609-4622.

Hadjihannas, M. V., Brückner, M., Jerchow, B., Birchmeier, W., Dietmaier, W. and Behrens, J. (2006). Aberrant Wnt/beta-catenin signaling can induce chromosomal instability in colon cancer. Proc. Natl. Acad. Sci. USA 103, 10747 10752.

Haigis, K. M., Caya, J. G., Reichelderfer, M. and Dove, W. F. (2002). Intestinal adenomas can develop with a stable karyotype and stable microsatellites. Proc. Natl. Acad. Sci. USA 99, 8927-8931.

Hamada, F., Tomoyasu, Y., Takatsu, Y., Nakamura, M., Nagai, S., Suzuki, A., Fujita, F., Shibuya, H., Toyoshima, K., Ueno, N. et al. (1999). Negative regulation of Wingless signaling by $\mathrm{D}$-axin, a Drosophila homolog of axin. Science 283, 1739-1742

Hanahan, D. and Weinberg, R. A. (2011). Hallmarks of cancer: the next generation. Cell 144, 646-674.

Hebert, A. M., DuBoff, B., Casaletto, J. B., Gladden, A. B. and McClatchey, A. I. (2012). Merlin/ERM proteins establish cortical asymmetry and centrosome position. Genes Dev. 26, 2709-2723.

Janssen, A., van der Burg, M., Szuhai, K., Kops, G. J. and Medema, R. H. (2011). Chromosome segregation errors as a cause of DNA damage and structural chromosome aberrations. Science 333, 1895-1898.

Jimbo, T., Kawasaki, Y., Koyama, R., Sato, R., Takada, S., Haraguchi, K. and Akiyama, T. (2002). Identification of a link between the tumour suppressor APC and the kinesin superfamily. Nat. Cell Biol. 4, 323-327.

Kao, L. R. and Megraw, T. L. (2009). Centrocortin cooperates with centrosomin to organize Drosophila embryonic cleavage furrows. Curr. Biol. 19, 937-942.

Kaplan, K. B., Burds, A. A., Swedlow, J. R., Bekir, S. S., Sorger, P. K. and Näthke, I. S. (2001). A role for the Adenomatous Polyposis Coli protein in chromosome segregation. Nat. Cell Biol. 3, 429-432.

Kawasaki, Y., Senda, T., Ishidate, T., Koyama, R., Morishita, T., Iwayama, Y., Higuchi, O. and Akiyama, T. (2000). Asef, a link between the tumor suppressor APC and G-protein signaling. Science 289, 1194-1197.

Lengauer, C., Kinzler, K. W. and Vogelstein, B. (1997). Genetic instability in colorectal cancers. Nature 386, 623-627.

Liu, X., Wu, H., Loring, J., Hormuzdi, S., Disteche, C. M., Bornstein, P. and Jaenisch, R. (1997). Trisomy eight in ES cells is a common potential problem in gene targeting and interferes with germ line transmission. Dev. Dyn. 209 85-91.

Liu, J., Xing, Y., Hinds, T. R., Zheng, J. and Xu, W. (2006). The third 20 amino acid repeat is the tightest binding site of APC for beta-catenin. J. Mol. Biol. 360 133-144.

Longo, L., Bygrave, A., Grosveld, F. G. and Pandolfi, P. P. (1997). The chromosome make-up of mouse embryonic stem cells is predictive of somatic and germ cell chimaerism. Transgenic Res. 6, 321-328.

Louie, R. K., Bahmanyar, S., Siemers, K. A., Votin, V., Chang, P., Stearns, T. Nelson, W. J. and Barth, A. I. (2004). Adenomatous polyposis coli and EB1 
localize in close proximity of the mother centriole and EB1 is a functional component of centrosomes. J. Cell Sci. 117, 1117-1128.

Madigan, J. P., Chotkowski, H. L. and Glaser, R. L. (2002). DNA double-strand break-induced phosphorylation of Drosophila histone variant H2Av helps prevent radiation-induced apoptosis. Nucleic Acids Res. 30, 3698-3705.

Mazars, G. R., Portier, M., Zhang, X. G., Jourdan, M., Bataille, R., Theillet, C. and Klein, B. (1992). Mutations of the p53 gene in human myeloma cell lines. Oncogene 7, 1015-1018.

Mazumdar, A. and Mazumdar, M. (2002). How one becomes many: blastoderm cellularization in Drosophila melanogaster. Bioessays 24, 1012-1022.

McCartney, B. M., Dierick, H. A., Kirkpatrick, C., Moline, M. M., Baas, A., Peifer, M. and Bejsovec, A. (1999). Drosophila APC2 is a cytoskeletallyassociated protein that regulates wingless signaling in the embryonic epidermis. J. Cell Biol. 146, 1303-1318

McCartney, B. M., McEwen, D. G., Grevengoed, E., Maddox, P., Bejsovec, A. and Peifer, M. (2001). Drosophila APC2 and Armadillo participate in tethering mitotic spindles to cortical actin. Nat. Cell Biol. 3, 933-938.

McCartney, B. M., Price, M. H., Webb, R. L., Hayden, M. A., Holot, L. M., Zhou, M., Bejsovec, A. and Peifer, M. (2006). Testing hypotheses for the functions of APC family proteins using null and truncation alleles in Drosophila. Development 133, 2407-2418.

Migliore, L., Migheli, F., Spisni, R. and Coppedè, F. (2011). Genetics, cytogenetics, and epigenetics of colorectal cancer. J. Biomed. Biotechnol. 2011, 792362.

Minestrini, G., Harley, A. S. and Glover, D. M. (2003). Localization of Pavarotti$\mathrm{KLP}$ in living Drosophila embryos suggests roles in reorganizing the cortical cytoskeleton during the mitotic cycle. Mol. Biol. Cell 14, 4028-4038.

Näthke, I. (2006). Cytoskeleton out of the cupboard: colon cancer and cytoskeletal changes induced by loss of APC. Nat. Rev. Cancer 6, 967-974.

Phelps, R. A., Broadbent, T. J., Stafforini, D. M. and Jones, D. A. (2009). New perspectives on APC control of cell fate and proliferation in colorectal cancer. Cell Cycle 8, 2549-2556.

Radulescu, S., Ridgway, R. A., Appleton, P., Kroboth, K., Patel, S., Woodgett, J., Taylor, S., Nathke, I. S. and Sansom, O. J. (2010). Defining the role of APC in the mitotic spindle checkpoint in vivo: APC-deficient cells are resistant to Taxol. Oncogene 29, 6418-6427.

Rajagopalan, H., Nowak, M. A., Vogelstein, B. and Lengauer, C. (2003). The significance of unstable chromosomes in colorectal cancer. Nat. Rev. Cancer $\mathbf{3}$ 695-701.

Roberts, D. M., Pronobis, M. I., Poulton, J. S., Waldmann, J. D., Stephenson, E. M., Hanna, S. and Peifer, M. (2011). Deconstructing the ßcatenin destruction complex: mechanistic roles for the tumor suppressor APC in regulating Wnt signaling. Mol. Biol. Cell 22, 1845-1863.

Roberts, D. M., Pronobis, M. I., Poulton, J. S., Kane, E. G. and Peifer, M (2012). Regulation of Wnt signaling by the tumor suppressor adenomatous polyposis coli does not require the ability to enter the nucleus or a particular cytoplasmic localization. Mol. Biol. Cell 23, 2041-2056.

Rogers, S. L., Rogers, G. C., Sharp, D. J. and Vale, R. D. (2002). Drosophila EB1 is important for proper assembly, dynamics, and positioning of the mitotic spindle. J. Cell Biol. 158, 873-884.

Rusan, N. M. and Peifer, M. (2008). Original CIN: reviewing roles for APC in chromosome instability. J. Cell Biol. 181, 719-726.
Rusan, N. M., Akong, K. and Peifer, M. (2008). Putting the model to the test: are APC proteins essential for neuronal polarity, axon outgrowth, and axon targeting? J. Cell Biol. 183, 203-212.

Sakurai, H., Okado, M., Ito, F. and Kawasaki, K. (2011). Anaphase DNA bridges induced by lack of RecQ5 in Drosophila syncytial embryos. FEBS Lett. $\mathbf{5 8 5}$ 1923-1928.

Sarpal, R., Todi, S. V., Sivan-Loukianova, E., Shirolikar, S., Subramanian, N. Raff, E. C., Erickson, J. W., Ray, K. and Eberl, D. F. (2003). Drosophila KAP interacts with the kinesin II motor subunit KLP64D to assemble chordotonal sensory cilia, but not sperm tails. Curr. Biol. 13, 1687-1696.

Seeling, J. M., Miller, J. R., Gil, R., Moon, R. T., White, R. and Virshup, D. M. (1999). Regulation of beta-catenin signaling by the B56 subunit of protein phosphatase 2A. Science 283, 2089-2091.

Shih, I. M., Zhou, W., Goodman, S. N., Lengauer, C., Kinzler, K. W. and Vogelstein, B. (2001). Evidence that genetic instability occurs at an early stage of colorectal tumorigenesis. Cancer Res. 61, 818-822

Sieber, O. M., Heinimann, K., Gorman, P., Lamlum, H., Crabtree, M., Simpson, C. A., Davies, D., Neale, K., Hodgson, S. V., Roylance, R. R. et al. (2002). Analysis of chromosomal instability in human colorectal adenomas with two mutational hits at APC. Proc. Natl. Acad. Sci. USA 99, 16910-16915.

Silkworth, W. T., Nardi, I. K., Paul, R., Mogilner, A. and Cimini, D. (2012). Timing of centrosome separation is important for accurate chromosome segregation. Mol. Biol. Cell 23, 401-411.

Smits, R., Kielman, M. F., Breukel, C., Zurcher, C., Neufeld, K., JagmohanChangur, S., Hofland, N., van Dijk, J., White, R., Edelmann, W. et al. (1999). Apc1638T: a mouse model delineating critical domains of the adenomatous polyposis coli protein involved in tumorigenesis and development. Genes Dev. 13, 1309-1321.

Sudhaharan, T., Goh, W. I., Sem, K. P., Lim, K. B., Bu, W. and Ahmed, S. (2011). Rho GTPase Cdc42 is a direct interacting partner of Adenomatous Polyposis Coli protein and can alter its cellular localization. PLOS ONE 6, e16603

Sullivan, W. (2009). Centrosomes: CNN's broadcast reaches the cleavage furrow. Curr. Biol. 19, R513-R515.

Takada, S., Kelkar, A. and Theurkauf, W. E. (2003). Drosophila checkpoint kinase 2 couples centrosome function and spindle assembly to genomic integrity. Cell 113, 87-99.

Tanenbaum, M. E. and Medema, R. H. (2010). Mechanisms of centrosome separation and bipolar spindle assembly. Dev. Cell 19, 797-806.

Thompson, S. L. and Compton, D. A. (2008). Examining the link between chromosomal instability and aneuploidy in human cells. J. Cell Biol. 180, 665672

Watanabe, T., Wang, S., Noritake, J., Sato, K., Fukata, M., Takefuji, M., Nakagawa, M., Izumi, N., Akiyama, T. and Kaibuchi, K. (2004). Interaction with IQGAP1 links APC to Rac1, Cdc42, and actin filaments during cell polarization and migration. Dev. Cell 7, 871-883.

Webb, R. L., Zhou, M. N. and McCartney, B. M. (2009). A novel role for an APC2-Diaphanous complex in regulating actin organization in Drosophila. Development 136, 1283-1293.

Zhou, M. N., Kunttas-Tatli, E., Zimmerman, S., Zhouzheng, F. and McCartney, B. M. (2011). Cortical localization of APC2 plays a role in actin organization but not in Wnt signaling in Drosophila. J. Cell Sci. 124, 1589-1600. 